\title{
Effects of Digital Filtering on the Classification Performance of Steady-State Visual Evoked Potential Based Brain-Computer Interfaces
}

\author{
V. ÇETIN, S. OZEKES and H.S. VAROL
}

\begin{abstract}
The electrical activity that occurs during the communication of neurons is recorded by a method called electroencephalography. Brain computer interfaces utilize various electrophysiological sources obtained from different regions of the brain. The electrophysiological source used in this study is the electrical activity seen in the occipital lobes as a result of visual stimuli that flicker at certain frequencies, and is called steady-state visual evoked potential. The main goal in this work is not to try to improve the classification performance but to investigate the effects of different digital filtering algorithms on classification performance. The effects of the high pass and low pass filtering on the classification performance in steady-state visual evoked potential based brain computer interfaces are inves tigated. As a result of this study, no significant change in the classification performances of designs with only high pass filtering, and high and low pass filtering, has been observed. In addition, it has been observed that only the designs include a high-pass filter implementation give better classification performance in many cases. Consequently, it is concluded that low-pass filtering in steady-state visual evoked potential based brain-computer interfaces does not provide the desired contribution to classification performance.
\end{abstract}

Index Terms - Brain-Computer Interfaces, Machine Learning, Biomedical Signal Processing, Digital Filters, Intelligent Systems.

\section{INTRODUCTION}

$\mathrm{E}$ VOKED ELECTRICAL signals, caused by a visual stimulus are called visual evoked potentials and are recorded from the occipital and parietal lobes of the brain. Stimuli of frequencies higher than $3.5 \mathrm{~Hz}$ are called steady-

VOLKAN ÇETIN, is with Department of Computer Engineering Istanbul Arel University, Istanbul, Turkey, (e-mail: volkancetin@ @arel.edu.tr).

(iD) https://orcid.org/0000-0003-3864-9246

SERHAT OZEKES, is with Department of Computer Engineering, Uskudar University, Istanbul, Turkey, (e-mail: serhat.ozekes@uskudar.edu.tr).

(iD https://orcid.org/0000-0002-2306-6008

HÜSEYIN SELÇUK VAROL, is with Department of Electronics and Communications Engineering Doğuş University, Istanbul, Turkey, (e-mail: hsvarol@dogus.edu.tr.com).

(iD https://orcid.org/0000-0002-3968-4230

Manuscript received December 02, 2019; accepted January 26, 2020. DOI: $\underline{10.17694 / \text { bajece. } 654288}$ state visual evoked potentials (SSVEP) because they produce a quasi- sinusoidal oscillation at the same frequency as the stimulus due to overlapping of excited action potentials [1]. SSVEP is best observed when the stimulus frequency is approximately $15 \mathrm{~Hz}$ while a light source that stimulates the retina at any frequency between $3.5 \mathrm{~Hz}$ and $75 \mathrm{~Hz}$ generates a signal of the same frequency in the visual cortex[2].

Researchers studied on various classification problems using the SSVEP-based BCI systems. Lalor et al. showed that control of computer games can be performed using brain signals with a SSVEP-based BCI system [3]. They applied a binary classification on EEG acquired from $\mathrm{O} 1$ and $\mathrm{O} 2$ electrodes, and used two stimuli with frequencies of $6 \mathrm{~Hz}$ and $25 \mathrm{~Hz}$. They applied a spectral power density (SPD)-based classification, and used the arithmetic mean of the SPD values obtained from $\mathrm{O} 1$ and $\mathrm{O} 2$ electrodes as features. Kelly et al. performed a left and right classification on EEG obtained from $\mathrm{O} 1$ and $\mathrm{O} 2$ electrodes using two stimuli at frequencies of 10 $\mathrm{Hz}$ and $12 \mathrm{~Hz}$ [4]. They applied a SPD-based classification algorithm. Muller-Putz and Pfurtscheller demonstrated that a four-task classification of a biaxial hand prosthesis by using a SSVEP-based BCI that makes it possible the use of BCI based neuro-prosthesis in situations such as spinal cord injury [5]. Prueckl and Guger developed a four-task classification of forward, backward, right and left with a SSVEP-based BCI using four stimuli at $10 \mathrm{~Hz}, 11 \mathrm{~Hz}, 12 \mathrm{~Hz}$ and $13 \mathrm{~Hz}$ frequencies [1]. They used the first and second harmonics obtained from the frequency spectrum of the SSVEP. They acquired the EEG from $\mathrm{O} 1, \mathrm{O} 2, \mathrm{Oz}, \mathrm{PO} 3, \mathrm{PO} 4, \mathrm{PO} 7, \mathrm{PO} 8$ and $\mathrm{POz}$ electrodes, and perform a SPD-based classification. Bin et al. designed a SSVEP-based BCI system by performing a canonical correlation based classification on EEG [6]. They acquired EEG from nine channels from occipital and temporal regions of the brain. Luo and Sullivan performed a four-task classification of SSVEP by using only the PO2 electrode [2]. They applied a SPD-based classification, and used four SSVEP stimuli at $9 \mathrm{~Hz}, 10 \mathrm{~Hz}, 11 \mathrm{~Hz}$ and $12 \mathrm{~Hz}$ frequencies. Volosyak developed a SSVEP-based spelling system with a classification of six tasks Based on the five different SSVEP stimuli at frequencies $6.67 \mathrm{~Hz}, 7.5 \mathrm{~Hz}, 8.57 \mathrm{~Hz}, 10 \mathrm{~Hz}$ and 12 
$\mathrm{Hz}$ [7]. Long et al. performed a battery-powered chair control using a hybrid BCI approach [8]. While using the sensorimotor based EEG for the right-left rotation and deceleration of the chair, they used the SSVEP signals obtained from $\mathrm{O} 1, \mathrm{O} 2$ and $\mathrm{Oz}$ electrodes for acceleration. Lee et al. controlled a mobile robot using SSVEP stimuli at $13 \mathrm{~Hz}$, $14 \mathrm{~Hz}$ and $15 \mathrm{~Hz}$ frequencies, corresponding to three different orientations as forward, right and left [9]. Zhang et al. designed an SSVEP-based BCI using canonical correlationbased classification, and stimuli at $6 \mathrm{~Hz}, 7 \mathrm{~Hz}, 8 \mathrm{~Hz}$ and $9 \mathrm{~Hz}$ frequencies. They obtained EEG from the electrodes O1, O2, $\mathrm{Oz}, \mathrm{P} 7, \mathrm{P} 8, \mathrm{P} 3, \mathrm{P} 4$ and Pz [10].

In general, stimuli that oscillate at frequencies higher than a certain threshold value are called critical vibration frequency (CVF). Frequencies in the range of $50 \mathrm{~Hz}$ to $60 \mathrm{~Hz}$ are perceived as not vibrating by the visual cortex. This situation results in less eye fatigue in subjects [11]. Sakurada et al. designed a BCI system in which stimuli were used at frequencies above the CVF [11]. The frequencies of $41 \mathrm{~Hz}, 43$ $\mathrm{Hz}$ and $45 \mathrm{~Hz}$ are used as stimuli that are below CVF, and 61 $\mathrm{Hz}, 63 \mathrm{~Hz}$ and $65 \mathrm{~Hz}$ as stimuli above the CVF. They compared the classification results of the system. Although frequencies above the CTF had a slight decline in the classification performance of the BCI, subjects reported less visual fatigue in stimuli at these frequencies. They have shown that high frequency visual stimuli above the CVF can be used in the design of SSVEP-based BCI systems. In previous years several studies has also been conducted to show the connection of the classification performance of EEG and the volume of music, [12] the effect of stimuli frequencies on eyes[11] and the analysis of the performance of different age groups [13]. On the other hand, there is no study has been observed in the literature showing the connection between classification performance and digital filtering algorithms in SSVEP-based BCI applications.

Brain-computer interface (BCI) design usually consists of signal processing, feature extraction and classification stages. In the signal processing stage, it is aimed to improve the signal quality through digital filtering. On the other hand, Widmann et al. have come to the conclusion that digital filtering should not be regarded as an essential step in EEG studies [14]. Vanrullen has reported that data associated with an event related activity can spread over hundreds of milliseconds on EEG due to filtering, and that can cause problems in assessing the timing and dynamics of brain activities [15]. He even advised not to apply low pass filtering when possible. The question in this case is the acceptability of decreasing classification performance, if any, versus decreasing time complexity of the filtering algorithm, since no digital filtering or only high pass filtering is applied. This study tries to answer this question by comparing the classification performances in cases where certain conventional digital filtering methods are used and not used.

\section{MATERIALS AND METHODS}

EEG recordings obtained from three subjects were used in this study. The stimulus frequencies, each corresponding to a separate task, are given in Table 1. A four-minute EEG was recorded for each task. Subjects were rested for three minutes between recordings. The user interface of the stimulus program is shown in Figure 1. The program consists of a maximum of 6 stimuli flickering at the desired frequencies. The distances of the flickers to each other and to the edges of the monitor are shown in Figure 1. These distances were measured on a 21.5" monitor when the program was full screen. During the EEG recordings the distance from the monitor to the subjects is set to be approximately $60 \mathrm{~cm}$.

TABLE I

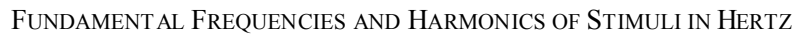

\begin{tabular}{|c|c|c|c|c|}
\hline Stimuli & $\begin{array}{c}1^{\text {st }} \\
\text { Harmonic }\end{array}$ & $\begin{array}{c}2^{\text {nd }} \\
\text { Harmonic }\end{array}$ & $\begin{array}{c}3^{\text {rd }} \\
\text { Harmonic }\end{array}$ & $\begin{array}{c}4^{\text {th }} \\
\text { Harmonic }\end{array}$ \\
\hline 1 & 4 & 8 & 12 & 16 \\
\hline 2 & 4.6 & 9.1 & 13.7 & 18.2 \\
\hline 3 & 5.3 & 10.6 & 15.9 & 21.2 \\
\hline 4 & 6.4 & 12.7 & 19.1 & 25.5 \\
\hline
\end{tabular}

A neuro-headset, manufactured by Emotiv, was used for EEG recordings. There are 16 electrodes on the headset, two of which are reference electrodes. The active electrodes in the neuro-headset are in fixed positions as AF3, F7, F3, FC5, T7, $\mathrm{P} 7, \mathrm{O} 1, \mathrm{O} 2, \mathrm{P} 8, \mathrm{~T} 8, \mathrm{FC} 6, \mathrm{~F} 4, \mathrm{~F} 8, \mathrm{AF} 4 . \mathrm{P} 3$ and P4 are used as reference electrodes. In this study, EEG signals obtained from occipital lobes were required because a SSVEP- based BCI design was analyzed. Therefore, only the data obtained from the channels $\mathrm{O} 1$ and $\mathrm{O} 2$ are used. 


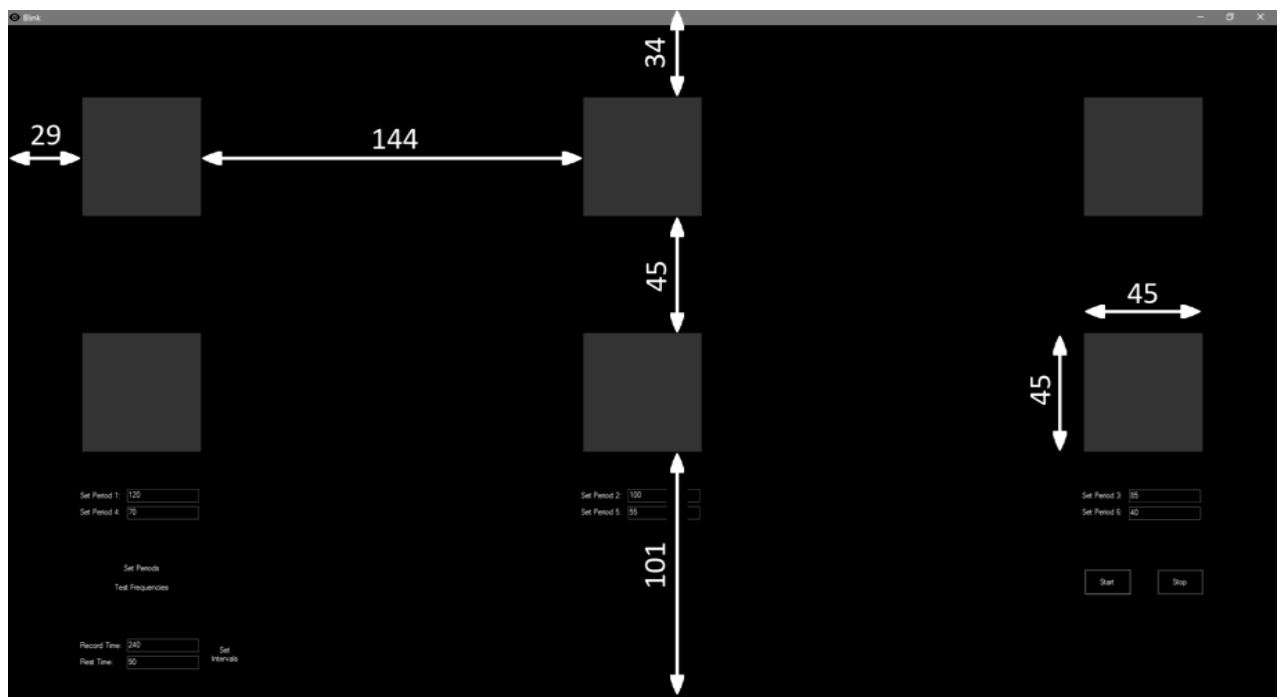

Fig. 1. User Interface of the Stimulus Program, and distances of the flickers to each other and to the edges of the monitor

\section{A. Digital Filter Characteristics}

Butterworth infinite impulse response (IIR) filters or finite impulse response (FIR) filters are usually applied in electrophysiology [14]. Generally, two parameters are taken into consideration in IIR or FIR filter design preference. These parameters are system stability and linear phase. IIR filters are not always stable due to feedback. On the other hand, FIR filters are always stable because their design does not include any feedback. In addition, symmetric FIR filters always have a linear phase. When evaluated in terms of system stability and linear phase, the superiority of FIR filters over IIR filters is evident. The advantage of IIR filters over FIR filters is that the desired filter characteristics can be achieved with low-order filters. In this study, FIR filters were applied to avoid possible signal distortions due to non-linear phase. In addition, FIR filter designs, which the filter length is usually relatively longer, are thought to serve better for the purpose of this study. For instance, a 40th-order filter causes more time delay than a 5th-order filter. Thus, the effect of a significant time delay on the classification performance can be better observed by applying a 40th-order filter. Figure 2and Figure 3 show the frequency (black curve) and phase (grey line) spectra of highpass (HPF) and low-pass (LPF) filters applied on EEG signals in this study.

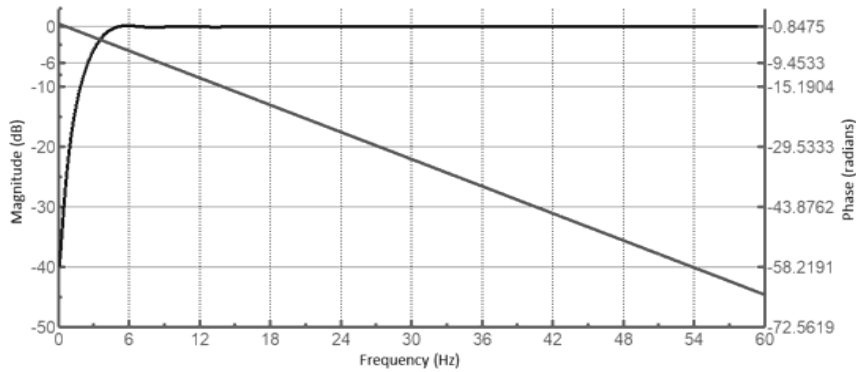

Fig. 2. Frequency and phase spectra of high pass filter applied on EEG data

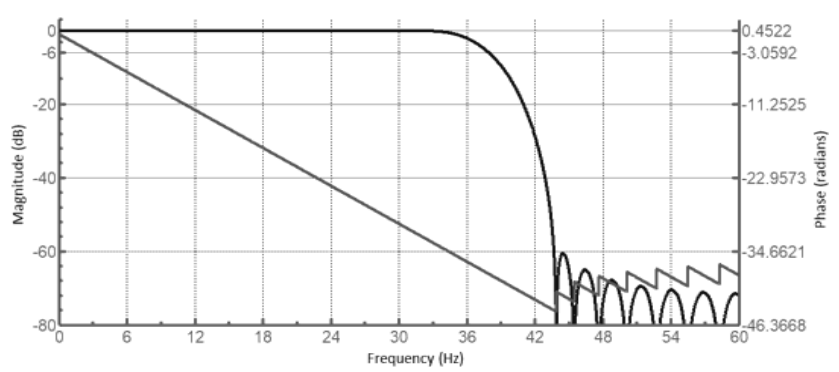

Fig. 3. Frequency and phase spectra of low pass filter applied on EEG data

As one approaches the ideal filter characteristics, the time delay resulting from filtering increases. The time delay of a filter is given by

$$
\Delta t=\frac{N-1}{2} T
$$

where $N$ is the filter length and $T$ is the sampling period. The HP and LP filters applied in this study have $172 \mathrm{~ms}$ and $160 \mathrm{~ms}$ time delays respectively. 
Filter length is an important parameter, especially when the time windows are relatively shorter. When a signal sampled at $128 \mathrm{~Hz}$ is analyzed in two-second windows, each piece of data segment consists of 256 samples. Thus, the HPF with a length of 45 makes the first 22 samples invalid which is $8.6 \%$ of the data, as shown in Figure 4(a). This is the edge effect created by the filter on the edges of the data. The edge effect caused by two filters with lengths 79 and 171 can be observed in Figure 4(b) and Figure 4(c), respectively. As one approaches the ideal filter characteristics, the edge effect due to the increase of the filter length causes a significant data loss which cannot be accepted especially in real time applications.
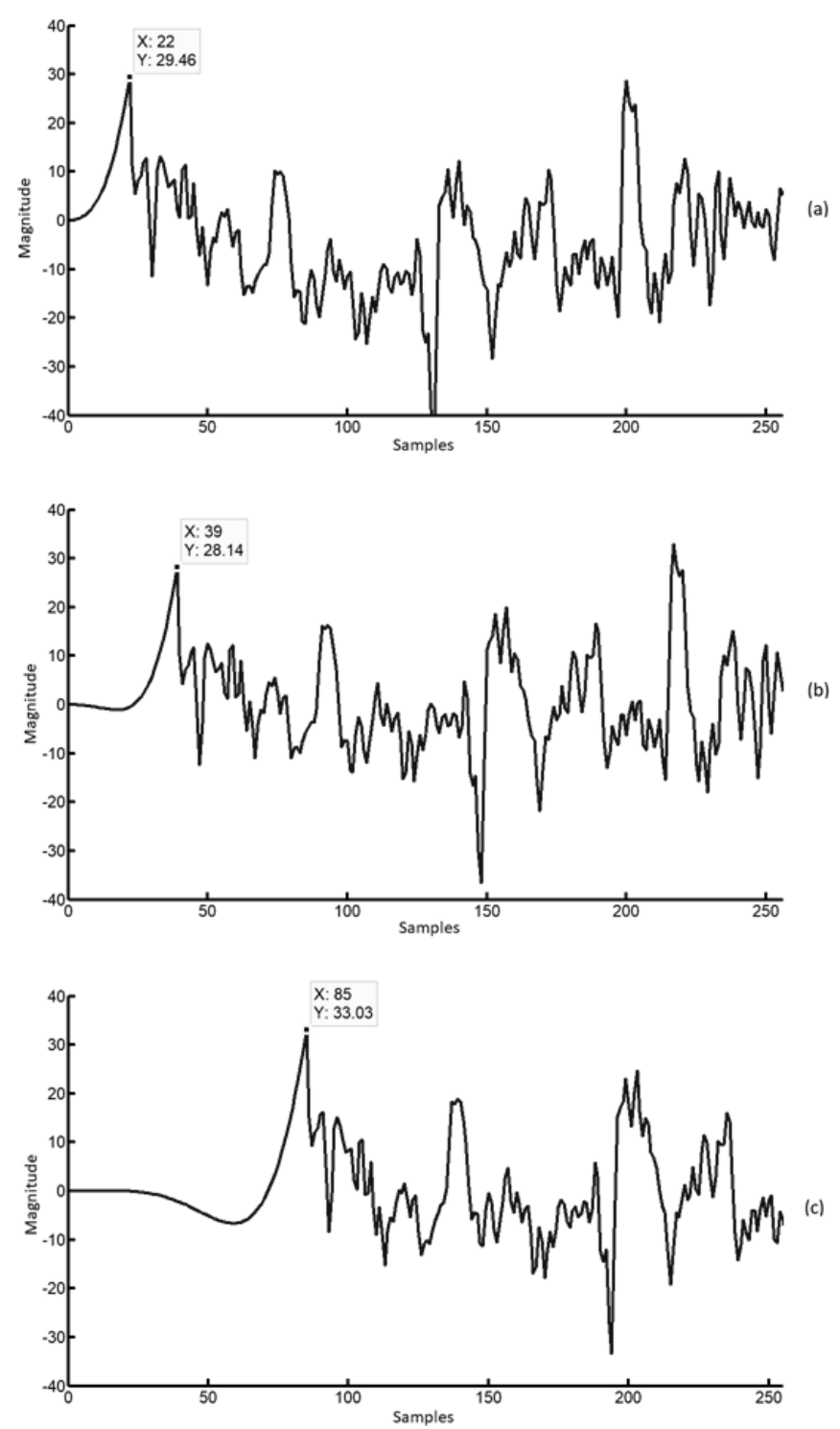

Fig. 4. Edge effect due to filters with lengths of (a) 45, (b) 79 and (c) 171, respectively

\section{B. Classification}

In general, it is observed that the most widely used classification methods in the BCI literature are artificial neural networks (ANN), support vector machines (SVM) and Naive Bayes (NB) $[16,17]$. In this study, a three layered artificial neural network model was used for classification.

SSVEP-based BCI users are best at modulating the spectral properties of the stimuli. Therefore, the spectral properties of EEG signals are mostly used as differentiating features for classification in SSVEP-based BCI applications [1,18-23]. For this reason the feature vector consists of the total and relative band power values of the first and second harmonics obtained from the frequency spectra of the SSVEPs. In this study, binary, ternary and quaternary classifications were performed. Three-layered perceptron network, trained by backpropagation algorithm, was used in classification. The input and the output layers contain neurons as many as the number of features and number of classes, respectively. Number of neurons in hidden layer is set to be the mean of the number of neurons in the input and the output layers. The learning rate of the network is 0.3 and the training iteration is 40 . A 10 -fold cross-validation model was used to evaluate the classification results. Each fold is composed of random samples with equal class distribution.

\section{RESULTS}

Four different cases were investigated in this study. These are cases which no digital filtering is applied, only HPF is applied, only LPF is applied, and HPF and LPF are applied sequentially. The applied HPF and LPF characteristics are given in Table 2.

TABLE II

HPF AND LPF CHARACTERISTICS APPLIED IN FILTERING EEG SignalS

\begin{tabular}{|l|c|c|}
\hline & HPF & LPF \\
\hline Attenuation & $37 \mathrm{~dB}$ & $60.5 \mathrm{~dB}$ \\
\hline Passband Ripple & $0.33 \mathrm{~dB}$ & $0.1 \mathrm{~dB}$ \\
\hline Transition Bandwidth & $4.6 \mathrm{~Hz}$ & $12 \mathrm{~Hz}$ \\
\hline $6 \mathrm{~dB}$ Point & $2.48 \mathrm{~Hz}$ & $38 \mathrm{~Hz}$ \\
\hline $3 \mathrm{~dB}$ Point & $3.22 \mathrm{~Hz}$ & $36.7 \mathrm{~Hz}$ \\
\hline Filter Length & 45 & 42 \\
\hline
\end{tabular}

The accuracy percentages of the binary, ternary and quaternary classifications using 2 and 4 second window lengths are given in Table 3. There are two important conclusions from Table 3. First, digital HPF increases classification performance by an average of $9.38 \%$ in all classifications of all subjects. The fact that the digital HPF improves classification performance in all cases, independent of time window, subject and number of classes, suggests that the electronic HPF on neuro-headset alone does not show adequate filtering performance for a BCI application. On the 
other hand, it is observed that the digital LPF does nothaveasignificanteffectontheclassificationperformanceandin mostcasesithasa negative effect. This can be explained by the fact that the electronic LPF in the neuro-headset is capable of providing adequate classification performance. Another explanation may be that the time delay and edge effect due to digital LPF have too much effect, while the low energy of high frequency noises has a less negative effect on classification performance.

TABLE III

The Accuracy Percentages of The Binary, Ternary and QUATERNARY ClaSSIFICATIONS USING 2 AND 4 SECOND WINDOW LENGTHS

$2 \mathrm{~s} \quad 4 \mathrm{~s}$

2Tasks 3Tasks 4Tasks 2 Tasks 3 Tasks 4 Tasks

\begin{tabular}{|c|c|c|c|c|c|c|c|}
\hline & No filtering & 68.75 & 55.28 & 46.04 & 75.83 & 59.44 & 46.67 \\
\hline \multirow[t]{4}{*}{ Subject 1} & Only LPF & 66.25 & 56.94 & 45.00 & 69.17 & 58.33 & 51.25 \\
\hline & Only HPF & 78.75 & 62.22 & 52.71 & 82.50 & 64.44 & 59.58 \\
\hline & $\mathrm{LPF}+\mathrm{HPF}$ & 78.75 & 57.78 & 49.17 & 78.33 & 67.22 & 60.83 \\
\hline & No filtering & 92.08 & 70.83 & 53.54 & 90.00 & 71.11 & 57.92 \\
\hline \multirow[t]{4}{*}{ Subject 2} & Only LPF & 90.00 & 67.78 & 51.67 & 85.00 & 61.67 & 61.67 \\
\hline & Only HPF & 96.67 & 85.83 & 66.46 & 94.17 & 82.22 & 55.42 \\
\hline & $\mathrm{LPF}+\mathrm{HPF}$ & 97.50 & 85.00 & 65.00 & 93.33 & 85.56 & 59.58 \\
\hline & No filtering & 71.25 & 56.11 & 51.25 & 81.67 & 64.44 & 57.08 \\
\hline \multirow[t]{4}{*}{ Subject 3} & 3 Only LPF & 73.33 & 54.17 & 51.46 & 79.17 & 59.44 & 55.83 \\
\hline & Only HPF & 82.92 & 69.22 & 67.92 & 87.50 & 75.00 & 74.58 \\
\hline & $\mathrm{LPF}+\mathrm{HPF}$ & 80.83 & 70.56 & 67.29 & 87.50 & 73.89 & 70.83 \\
\hline & No filtering & 77.36 & 60.74 & 50.28 & 82.50 & 65.00 & 53.89 \\
\hline \multirow[t]{3}{*}{ Mean } & Only LPF & 76.53 & 59.63 & 49.38 & 77.78 & 59.81 & 56.25 \\
\hline & Only HPF & 86.11 & 72.42 & 62.36 & 88.06 & 73.89 & 63.19 \\
\hline & $\mathrm{LPF}+\mathrm{HPF}$ & 85.69 & 71.11 & 60.49 & 86.39 & 75.56 & 63.75 \\
\hline
\end{tabular}

The second important conclusion is understood when comparing analyzes that includes applying only HPF and that includes applying HPF and LPF. Table 4 gives the average classification performances obtained from the aforementioned analyzes. It is seen that HPF and LPF together affected the classification performance at a range of $1.67 \%$ to $-1.87 \%$. Despite LPF-caused time delay, the classification performance increases up to $1.67 \%$ at most. In addition, it has been observed that the case which includes applying HPF and LPF together decreases the classification performance between $0.42 \%$ and $1.87 \%$ in certain cases. Results obtained from this study verify that the digital filtering is not an absolute step in BCI analysis as well as concluded by Widmann et al. for EEG analysis. It also verifies that LPF should not be applied unless there is a significant necessity as Vanrullen proposes.

TABLE IV

Comparison of Classification Performances of BCI Designs

\begin{tabular}{ccccccc}
\hline & \multicolumn{3}{c}{ 2s } & \multicolumn{3}{c}{ 4s } \\
\cline { 2 - 7 } & 2 Tasks & 3 Tasks & 4 Tasks & 2 Tasks & 3 Tasks & 4 Tasks \\
\hline Only HPF & 86.11 & 72.42 & 62.36 & 88.06 & 73.89 & 63.19 \\
\hline HPF + LPF & 85.69 & 71.11 & 60.49 & 86.39 & 75.56 & 63.75 \\
\hline Difference & -0.42 & -1.31 & -1.87 & -1.67 & 1.67 & 0.56
\end{tabular}

\section{CONCLUSION}

In this study, the effect of different digital filtering strategies on the classification performance of BCI applications is investigated. We have experimentally confirmed the conclusions and the suggestions with the adverse effects of the digital filtering in EEG analysis mentioned in previous publications. This study shows that low-pass filtering in BCI applications does not provide the desired contribution to classification performance. On the other hand when evaluating the results obtained from this study the characteristics of the EEG device and digital filters should not be overlooked. The neuro-headset has an HPF at cut-off frequency of $0.15 \mathrm{~Hz}$ and a LPF at cut-off frequency of $43 \mathrm{~Hz}$. Different EEG recorders could probably contain electronic filters with different characteristics or no electronic filters at all. Therefore there should be no definite conclusion that LPF should not be applied in BCI applications. Instead, the effect of digital filters on the classification performance should be investigated and a digital filtering strategy should be determined according to the results in BCI applications. Another conclusion can also be deduced that the electronic filters contained in the EEG recording devices should not be relied upon. Although the neuro-headset used in this study have a HPF at a cut-off frequency of $0.15 \mathrm{~Hz}$, an additional digital HPF has improved the classification results.

\section{References}

[1] R. Prueckl, C. Guger, "A Brain-Computer Interface Based onSteady State Visual Evoked Potentials for Controlling a Robot." 10th International Work-Conference on Artificial Neural Networks, vol.10, no. 12 June, Salamanca,Spain. 2009. doi:https://doi.org/10.1007/978-3642-02478-8_86

[2] A.Luo, T.J. Sullivan, "A User-Friendly SSVEP-Based Brain-Computer Interface using a Time-Domain Classifier." Journal of Neural Engineering, vol. 7, no. 2, pp. 026010,2010. doi:10.1088/17412560/7/2/026010

[3] E.C. Lalor, S.P. Kell, C. Finucane, R. Burke, R. Smith, R.B. Reilly, G. McDarby, "Steady-State VEP-Based Brain-Computer Interface Control in an Immersive 3D Gaming Environment." EURASIP Journal on Applied Signal Processing, vol. 2005, no. 19, pp. 3156-3164,2005. doi: https://doi.org/10.1155/ASP.2005.3156

[4] S.P.Kelly, E.C. Lalor, C. Finucane, G. McDarby, R.B. Reilly, "Visual Spatial Attention Control in an Independent Brain-Computer Interface." 
IEEE Transactions on Biomedical Engineering, vol. 52 np. 9, pp. 15881596, 2005. doi: 10.1109/TBME.2005.851510

[5] G.R. Muller-Putz, G. Pfurtscheller, "Control of an Electrical Prosthesis with an SSVEP-Based BCI." IEEE Transactions on Biomedical Engineering, vol. 55, no. 1, pp. 361-364, 2008. doi: 10.1109/TBME.2007.897815

[6] G. Bin, X. Gao, Z. Yan, B. Hong,S. Gao, "An Online Multi-Channel SSVEP-Based Brain-Computer Interface using a Canonical Correlation Analysis Method. Journal of Neural Engineering", vol. 6, no. 4, pp. 046002, 2009. doi: 10.1088/1741-2560/6/4/046002

[7] I. Volosyak, "SSVEP-Based Bremen-BCI Interface - Boosting Information Transfer Rates." Journal of Neural Engineering, vol. 8, no.3, pp. 036020, 2011. doi: 10.1088/1741-2560/8/3/036020

[8] J. Long, Y.Li, H. Wang, T. Yu., J. Pan, F. Li, "A Hybrid Brain Computer Interface to Control the Direction and Speed of a Simulated or Real Wheelchair." IEEE Transactions on Neural Systems and Rehabilitation Engineering, vol. 20, no. 5, pp. 720-729, 2012. doi: 10.1109/TNSRE.2012.2197221

[9] P. Lee, H. Chang, T. Hsieh, H. Deng, C. Sun,“A Brain-Wave- Actuated Small Robot Car using Ensemble Empirical Mode DecompositionBased Approach." IEEE Transactions on Systems, Man, and Cybernetics - Part A: Systems And Humans, vol. 42, no. 5, pp. 10531064, 2012. doi:10.1109/TSMCA.2012.2187184

[10] Y. Zhang, G. Zhou, J. Jin, X. Wang, A. Cichocki, "SSVEP Recognition using Common Feature Analysis in Brain-Computer Interface.”Journal of Neuroscience Methods, vol. 244, pp. 8-15, 2015. doi: 10.1016/j.jneumeth.2014.03.012

[11] T. Sakurada, T. Kawase, T. Komatsu, K. Kansaku, "Use of HighFrequency Visual Stimuli Above the Critical Flicker Frequency in a SSVEP Based BMI." Clinical Neurophysiology, vol. 126, no. 10, pp. 1972-1978, 2015. doi: 10.1016/j.clinph.2014.12.010

[12] K. Hasan, S. Hossain, T.K. Ghosh, M. Ahmad, "A SSVEP Based EEG Signal Analysis to Discriminate the Effects of Music Levels on Executional Attention." American Journal of Bioscience and Bioengineering, vol. 3, no. 3-1, pp. 27-33, 2015. doi: 10.11648/j.bio.s.2015030301.15

[13] F. Gembler, P.Stawicki, I.Volosyak, "A Comparison of SSVEP-Based BCI-Performance Between Different Age Groups." 13th International Work-Conference on Artificial Neural Networks, 10-12 June, Palma de Mallorca, Spain, 2015. doi: https://doi.org/10.1007/978-3-319-19258$1 \_6$

[14] A. Widmann, E. Schröger, B. Maess, "Digital Filter Design for Electrophysiological Data - A Practical Approach. Journal of Neuroscience Methods", vol.250, pp. 34-46, 2014. doi: 10.1016/j.jneumeth.2014.08.002

[15] R. Vanrullen, "Four Common Conceptual Fallacies in Mapping the Time Course of Recognition." Frontiers in Psychology, vol. 2, Article365, 2011.doi: 10.3389/fpsyg.2011.00365

[16] L.F. Nicolas-Alonso, J. Gomez-Gil, "Brain Computer Interfaces, a Review." Sensors, vol. 12, no. 2, pp. 1211-1279, 2012. doi: $10.3390 / \mathrm{s} 120201211$

[17] F. Lotte, M. Congedo, A. Lecuyer, F. Lamarche, B. Arnaldi, "A Review of Classification Algorithms for EEG-Based Brain-Computer Interfaces." Journal of Neural Engineering, vol. 4 no. 2, R1, 2007. doi: 10.1088/1741-2552/aab2f2

[18] I. Volosyak, "SSVEP-Based Bremen-BCI Interface - Boosting Information Transfer Rates." Journal of Neural Engineering, vol. 8, no. 3, pp. 036020, 2011. doi: 10.1088/1741-2560/8/3/036020

[19] E.C. Lalor, S.P. Kelly, C. Finucane, R. Burke, R. Smith, R.B. Reilly, G. McDarby, "Steady-State VEP-Based Brain-Computer Interface Control in an Immersive 3D Gaming Environment."EURASIP Journal on Applied Signal Processing, vol. 2005 no. 19, pp. 3156-3164, 2005. doi:10.1155/ASP.2005.3156

[20] P. Lee, H. Chang, T. Hsieh, H. Deng, C. Sun, "A Brain-WaveActuated Small Robot Car using Ensemble Empirical Mode Decomposition-Based Approach." IEEE Transactions on Systems, Man, and Cybernetics -PartA: Systems And Humans, vol. 42, no. 5, pp. 1053-1064, 2012. doi: 10.1109/TSMCA.2012.2187184

[21] J.R. Wolpaw, E.W. Wolpaw, "Brain-Computer Interfaces Principles and Practice, Oxford University Press, Inc.”, New York, USA, 2012. doi: 10.1093/acprof:oso/9780195388855.001.0001

[22] Y. Wang, R. Wang, X. Gao, B. Hong, S. Gao, "A Practical VEP- Based Brain-Computer Interface." IEEE Transactions on Neural Systems and Rehabilitation Engineering, vol. 14, no. 2, 234-239, 2006. doi: 10.1109/TNSRE.2006.875576
[23] H. Gollee, I. Volosyak, A.J. McLachlan, K.J. Hunt, A. Graser, "An SSVEP-Based Brain-Computer Interface for the Control of Functional Electrical Stimulation.” IEEE Transactions on Biomedical Engineering, vol. 57, no. 8, pp. 1847-1855, 2010. doi:10.1109/TBME.2010.2043432

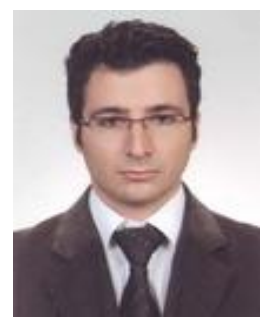

\section{BIOGRAPHIES}

VOLKAN ÇETIN was born in Burdur in 1984. He is a Ph.D. candidate in Institute of Pure and Applied Sciences of Marmara University. He gives lectures in Computer Engineering Department of Istanbul Arel University and Computer Engineering Department of Istanbul Sabahattin Zaim University.

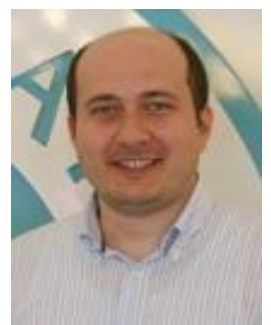

SERHAT OZEKES was born in Washington D.C., USA in 1978. He received his B.S., M.S. and Ph.D. degrees from Marmara University, Computer and Control Education Department in 2000, 2002 and 2006, respectively. He has published more than 25 papers in various international journals and conference proceedings on data mining, image processing and EEG signal processing.

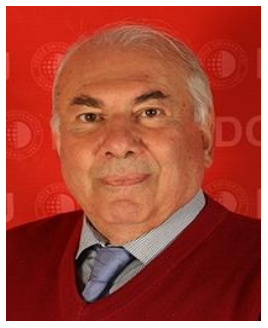

HÜSEYIN SELÇUK VAROL was born in Ankara in 1948. He received his Bachelor's and Master's degrees from the Middle East Technical University Physics Department in 1969 and 1972 respectively. $\mathrm{He}$ completed his $\mathrm{PhD}$ in Physical Electronics at Surrey University in England, and worked in many companies, universities and research institutions. He has been working at the Department of Electronics and Communications Engineering at Doğuş University, where he has been working since 2015. He has over 80 international and national publications, and his current area of scientific interest is biomedical image and signal processing. 